\title{
Deposition of Metallic Coatings on Polymer Surfaces using ${ }^{1}$ Cold Spray
}

\author{
R. Lupoi and W. O’ Neill \\ Institute for Manufacturing, Department of Engineering, University of Cambridge \\ 17 Charles Babbage Road, Cambridge, CB3 OFS, UK
}

\author{
Corresponding author is R. Lupoi \\ e-mail: r1372@cam.ac.uk \\ phone: (+44)01223764617 fax: (+44)01223464217
}

\begin{abstract}
Current coating technologies such as plasma spray, High Velocity Oxy Fuel (HVOF) or laser cladding involve the delivery of molten materials during the deposition process. However, such processes are not well suited to the deposition of metallic coatings on polymers and composites. ${ }^{1}$ Cold Spray (CS) has attracted much industrial interest over the past two decades. In this method, a material in powder form is accelerated on passage through a converging-diverging nozzle to high speeds via a high pressure coaxial carrier gas jet. The high impact kinetic energy deforms the particles, which creates effective bonding to the substrate.

This paper presents the results of an initial study on the potential of the ${ }^{1} \mathrm{CS}$ process to produce metallic coatings on non-metallic surfaces such as polymers and composites for engineering applications. Experimental and Computational Fluid Dynamics (CFD) results when spraying copper, aluminium and tin powder on a range of substrates such as PC/ABS, polyamide-6, polypropylene, polystyrene and a fibre-glass composite material are presented and analyzed.
\end{abstract}

KEYWORDS: Cold Spray, Deposition, Plastic coatings, Polymers, Composites, CFD. 


\section{1-INTRODUCTION}

There is a high demand for the production of metallic coatings in various industrial sectors, from simple engineering application such aesthetic layers to more complex applications like electronic devices. Coatings of various types are frequently laid on mechanical components to protect against hostile environments, or on instruments such as machining tools in such a way to enhance their performance and durability. When considering the manufacturing of metallic coatings on surfaces, thermal spray methods are widely used for industrial applications [1]. In such processes, the feedstock metal, typically in the form of powder or wire, is heated to melting point and propelled as individual droplets towards the working surface. The energy required for the melting process is usually generated by combustible gases or an electric arc. As the particles impinge on the substrate, they splat (deform) and solidify. Due to the nature of this process, coatings typically exhibit high levels of porosity caused by the incomplete bonding of the sprayed material, especially when processing course powders [2]. Coatings may also exhibit high levels of oxidation due to the in-flight entrainment of oxygen from ambient air [3]. A variant to the standard thermal spray is the High Velocity Oxygen Fuel (HVOF) process [4]. In this case, the combustion of several types of fuel (kerosene, acetylene or propylene) generate a hot high pressure gas stream in which powder is injected and accelerated through the centreline of the nozzle to be deposited on the substrate. An alternative coating method is ${ }^{1}$ Cold Spray (CS) [5]. In this case the feedstock powder is not melted, but only accelerated to high velocities through gases travelling at supersonic speed, typically nitrogen, air or helium, delivered by a converging-diverging type nozzle. On particle impact with the substrate, the high kinetic energy of the particles results in severe plastic deformation and the creation of a deposition layer. CS coatings typically show high bond strength and excellent mechanical characteristics, including metals of strong engineering interest such as aluminium, titanium and their alloys [6,7,8,9]. The deposition of thermoplastic powder has also been achieved onto metallic and non-metallic substrates [10]. 
Bray et al. have further improved the process by adding thermal energy to the deposition zone with the use a laser source, in such a way to reduce particle yield stresses, facilitate greater bond strengths and promote higher coating densities for lower particle kinetic energies [11].

Of particular interest for engineering applications are metallic coatings on surfaces such as plastics, fabrics or composite materials. The nature of such substrate makes the process of metal deposition particularly difficult. Successful coatings have been achieved with thermal spray through complete melting of the feedstock material. As an example, ${ }^{5}$ Voyer et al. have successfully managed to deposit aluminium on fabric materials by using flame spray [12]. It was possible to achieve coating conductivity, despite the high level of internal porosity. Also, in this case it was necessary to incorporate in the system a cooling apparatus, to protect the fabric material from the high temperature molten sprayed metal. Spraying without cooling system was only possible at standoff distances in excess of $250 \mathrm{~mm}$, resulting in low deposition efficiencies. Nevertheless, the increasing demand for low energy, environmental friendly, efficient and low-cost processes is driving away research from current methods to alternative techniques such as Cold Spray (CS).

There are few reports of the application of CS in the production of metallic coatings on polymer substrates. Zhang et al. [13] have investigated the effect of substrate type when spraying aluminium powder $(99.9 \mathrm{wt} \%$, average size $=25 \mu \mathrm{m})$. The range of materials included ABS and glass. Results have shown that deposits on non-metallic surfaces were thin and not continuous; only relatively small particles $(<5 \mu \mathrm{m})$ were adhered and heavy erosion of the plastic substrate was observed. Lee et al. $[14,15]$ suggested that mixing the feedstock powder with ceramic material such as $\mathrm{Al}_{2} \mathrm{O}_{3}$ may facilitate the deposition of metals onto hard non-metallic surfaces. The authors concluded that the ceramic material forms craters on the substrate, therefore creating a favourable surface topology to initiate the deposition process. 
On the other hand, Sturgeon et al. [16] have successfully formed aluminium coatings of about $600 \mu \mathrm{m}$ thickness on Carbon Fibre Reinforced Polymer (CFRP) surfaces. In this case, helium was used as carrier gas, heated up to a temperature of $300^{\circ} \mathrm{C}$. A De-Laval convergingdiverging nozzle geometry was utilized, with an inlet pressure of 20bar. Results showed good deposition characteristics, although the authors noted that the types of reinforced polymers on which metal deposition was achievable required further studies.

This paper further investigates the use of the CS process to form metallic coatings on polymer surfaces. Metals of general engineering interest such as copper, aluminium and tin have been sprayed onto substrates including a commercial blend of polycarbonate and ABS (PC/ABS), polystyrene, polyamide-6, polypropylene and a glass fibre composite material.

\section{2- ${ }^{1}$ CS APPLICATION TO NON-METALLIC SUBSTRATES}

An in-house Cold Spray facility developed at the University of Cambridge was used for experimental testing. The CS system is shown in Figure 1.

\section{Figure 1}

A nitrogen gas source of maximum pressure 30bar is fed through a converging-diverging nozzle. ${ }^{2}$ The system does not incorporate a gas-heater, therefore spraying is carried out at room temperature. The nozzles are typically made out of tool steel, with a variable diverging section length (from $70 \mathrm{~mm}$ up to $180 \mathrm{~mm}$ ). Such devices are capable of generating a supersonic gas stream travelling at the speed region of $650 \mathrm{~m} / \mathrm{s}$. On the other hand, powder feedstock is injected above the nozzle throat using a commercial high pressure powder feeder (wheel delivery type, Praxair Inc. 1264HP). The high gas speed imposes a significant drag force on the powder, which can be accelerated at velocities in the excess of $500 \mathrm{~m} / \mathrm{s}$ towards 
the substrate to form the coating. However, the maximum achievable velocity is dependent on the powder material and size.

Figure 2

Figure 3

Figure 4

Powders materials and geometries typically used in engineering applications were tested in theses initial trials. Specifically, Figure 2, 3 and 4 show Scanning Electron Microscope (SEM) images of the powder feedstock used, i.e. commercial aluminium, copper and tin. The aluminium was acquired from the Aluminium Powder Company Ltd., $-53+10 \mu \mathrm{m}$ size distribution. On the other hand, the copper $(\mathrm{HC} \mathrm{Cu})$ and tin $(\mathrm{SC} 10)$ powders are from Sandvik Ospray Ltd., characterized by $-32+10 \mu \mathrm{m}$ size distribution. The aluminium powder was produced by the water atomization process, therefore it exhibited irregular prismatic geometries; on the other hand the copper and tin particles were gas atomized giving a more regular and spherical shape.

In order to study the effect of powder impact speed on deposition characteristics, the nozzle inlet pressure was varied across the range 5-30bar. As an example of such relationship, Figure 5 shows the calculated centreline velocity magnitude of a spherical particle $(20 \mu \mathrm{m}$ diameter) against the carrier gas (Nitrogen) inlet pressure, from a Computational Fluid Dynamic (CFD) analysis using Fluent Inc.(version 6.3.26). The supersonic nozzle was De-Laval type, with $2 \mathrm{~mm}$ and $6 \mathrm{~mm}$ throat and exit diameter respectively. Also, the converging and diverging sections of the nozzle were $30 \mathrm{~mm}$ and $180 \mathrm{~mm}$ long. The model was created and meshed with 
elements of nearly 20000. The "high-mach-number" drag law algorithm was used in this case [17], suitable for spherical high speed particles. Pressure-inlet and pressure-outlet boundary conditions were set at the inlet and outlet surfaces of the computational domain, and the k- $\varepsilon$ realizable turbulence model was used. The simulations were converged up to the second order discretization scheme, by following the density-based solver algorithm. The graph in the figure shows the calculated speeds at the nozzle throat and exit, for $20 \mu \mathrm{m}$ diameter particles of aluminium, copper and tin. It can be appreciated that at increasing values of inlet pressure the speed of the particles within the nozzle grows. Also when spraying at the pressure of 30 bar the particle velocity raises of about double than the value at 5bar nearby the throat, and increases of approximately $50 \%$ at the exit of the nozzle. In addition, aluminium is shown to travel faster in comparison to copper and tin, due to its low specific weight.

Developed commercial CFD packages are nowadays valuable instruments to predict particles and flow characteristics in cold spray applications. Nickel et al. [18] have implemented Fluent to model supersonic flow over a shock tube spraying device, and also used the "high-machnumber" algorithm to model the particles drag law. On the other hand, Bray et al. [11] have extensively compared metallic particle velocities by a CFD analysis with Fluent against experimental measurements. Despite commercial powders may not be regarded as perfectly spherical, it was observed that computational and experimental results were in close agreement $(<5 \%)$ at both low and higher nozzle inlet pressures.

Figure 5 


\section{2-Spray trials}

Experiments were carried out in order to identify best process parameters setting, on untreated commercial polycarbonate-ABS blend (PC/ABS - Cycoloy C1100HF), polystyrene (Nova 124N), polyamide-6 (DSM Akulon F136-C1), polypropylene (Basell Moplen HP561R) and a glass-fibre composite material. Figure 6 shows a close up picture of coatings obtained at 5 bar and 30bar nozzle inlet pressure when spraying copper powder onto PC/ABS and the glassfibre reinforced composite material. The supersonic nozzle used in this case was characterized by an internal De-Laval profile with a diverging section length of $180 \mathrm{~mm}$. The 5 bar coating on PC/ABS, shown in Figure 6a, was obtained with a distance nozzle exit - substrate, the Standoff distance SoD, of $40 \mathrm{~mm}$, substrate transverse speed of $8.3 \mathrm{~mm} / \mathrm{s}$ and by imposing a rotational speed of $15 \mathrm{rpm}$ to the powder feeder wheel. On the other hand, the optimum operational settings for the 5bar coating on the composite material (Figure 6b) were same as above, but a visible coating could only be observed at lower powder flow rates, i.e. with 5rpm wheel speed. Results from Figure $6 \mathrm{a}$ and $6 \mathrm{~b}$ shows that a relatively thin layer of metal can be deposited on the surface at the imposed process conditions. On the other hand, Figure 6c shows an example of a 30bar coating on PC/ABS, produced with a SoD of 40mm, transverse speed of $16.6 \mathrm{~mm} / \mathrm{s}$ and a feeder speed of $18 \mathrm{rpm}$. The $30 \mathrm{bar}$ coating on the composite material is shown in Figure 6d, obtained with $40 \mathrm{~mm}$ SoD, transverse speed of $8.3 \mathrm{~mm} / \mathrm{s}$ and $12 \mathrm{rpm}$ feeder speed. For these last two cases, the change of the parameters settings (at the fixed inlet pressure of 30bar) resulted in very small variations to the tracks shown in Figure $6 \mathrm{c}$ and $6 \mathrm{~d}$, however the best observable results were obtained with the settings presented in this paper.

Consequently, when the nozzle is fed by nitrogen at 30bar, it is observed that less metal is laid down in comparison with the lower pressure case. In general, it was demonstrated that more material can be deposited at lower inlet pressures. A possible explanation of such result is given by the coating analysis in Figure 7, which shows optical micrographs of the transverse 
cross-sectional profiles, where the substrate material is below the coating. At small inlet pressures, i.e. when low energy impacts occurs, a first layer of particles embeds below the surface, as shown in Figure $7 \mathrm{a}$ and $7 \mathrm{~b}$. Powder material impacting on top of this first layer does not adhere and recoils off the surface. Once the first layer is deposited, subsequent impacts do not carry sufficient energy to adhere to the lower metal layer due to their low speed. In this case the impact energy is only sufficient to penetrate the plastic surface, but not to form a solid metal-to-metal coating. Such behaviour suggests that once the metal seed layer is deposited, the deposition mechanism on subsequent layers is similar to the one observed for metallic surfaces. In fact, powder interactions with the plastic material can only occur on the first coating stratum.

\section{Figure 6}

When increasing the nozzle inlet pressure (i.e. the particles speed) in the attempt to form a solid coating, heavy erosion of the substrate occurs as shown in Figure 7c. Empty craters are clearly visible on the cross-section of the PC/ABS. In this particular case, although the particle speed is optimal for the copper to create a metal coating, the impact energy and related impact stresses transmitted from the particle to the substrate go beyond the material strength, hence heavy erosion takes place. Figure $7 d$ shows the same effect, when using a glass-fibre reinforced composite. Despite the reinforced characteristics of this material, broken fibres are visible.

Figure 7

In addition, an attempt was made to deposit the non-spherical aluminium powder from Figure 2 on pure polycarbonate. The nozzle inlet pressure was set to its maximum level of 30bar. In 
this case, particle impacts did not impose severe damage to the polymer surface and no deposition was achieved. This effect could be due to the relatively low density of aluminium, being incapable of generating enough impact energy with the substrate in comparison to other materials, i.e. erosion is negligible [19]. However, it was not possible to deposit aluminium in this case. A reason for this relies upon its relatively high critical velocity, in the region of $600 \mathrm{~m} / \mathrm{s}$ [20], which may be beyond the maximum achievable with the current cold spray system with no gas-heating implemented.

Experimental results when using copper and aluminium powders have suggested that the ideal material to obtain metal deposition on substrates made out of plastic or composite surfaces may be characterized by both low density and critical speed, in order to avoid erosion of the substrate and at the same time uneconomic arrangements capable of accelerating particles to high velocity levels. With this respect, experiments were carried out with tin powder. The mechanical characteristics of tin may propose this material to be more suitable to initialize a coating formation onto plastic substrates. Studies have demonstrated that tin particles can begin to deposit at velocities as low as $200 \mathrm{~m} / \mathrm{s}$ [20,21], approximately three times lesser than aluminium and half the speed of copper; while the tin density stands between the two. Experimental trials were carried out, and the deposition of this type of metal was successful. It was possible to optimize nozzle type and spraying conditions to obtain thin solid coatings made of the tin powder from Figure 4 on a variety of plastic substrates (PC/ABS, polyamide6, polypropylene and polystyrene), as shown in the close-up pictures in Figure 8. Specifically, a nozzle with a diverging section of $70 \mathrm{~mm}$ long was employed. Air at 30bar was used as carrier gas in this case. Being tin a relatively soft metal, clogging of the nozzle at correspondence of its throat may occur. In order to avoid this problem, powder was injected axially after the throat, through a low-pressure powder feeder (Powder Feed Dynamic Inc., MARK XV), screw-delivery type. Optimum coating conditions were achieved with a SoD of 
$103 \mathrm{~mm}$ and by setting the rotational speed of the screw feeder to 200rpm. Tin deposited well at the substrate transverse speed of $14 \mathrm{~mm} / \mathrm{s}$ in all cases. In order to better identify the coatings characteristics, cross-sections were cut and visualized with the optical microscope and pictures shown in Figure 9. It can be clearly seen that with all substrate materials considered in this paper it was possible to achieve coatings ${ }^{3}$ in the range between $45 \mu \mathrm{m}$ and $100 \mu \mathrm{m}$ thickness. Deposited particles can be clearly identified in Figure 9d, as the coating in this case was chemically etched.

Figure 8

Figure 9

Their final geometry, when compared to the initial shape, suggests that plastic deformation occurred and particles deposited one on top of the other to form a multi-layer coating geometry. These type of coatings had shown good ${ }^{5}$ electrical conductivity, without the necessity of any post-treatment or pre-treatment. ${ }^{3}$ Coatings thickness following one pass varied from $45 \mu \mathrm{m}$ on the PC/ABS and polystyrene materials to nearly $100 \mu \mathrm{m}$ on the polymamide- 6 substrate. The attempt of a second pass on top of the formed first layer of tin resulted in the destruction of the coating in all cases. Reasons and causes for such behaviour are currently under investigation. By using the spraying parameters as input, it was therefore possible to calculate throughout a CFD analysis the average deposition velocity of the tin particles to form the coatings shown in Figure 8 and 9. Such speed was computed to be $310 \mathrm{~m} / \mathrm{s}$, which stands within the theoretical deposition velocity range given by Schmidt for tin powder [19] on a metallic substrate. 


\section{3-Process Characterization}

Experimental results obtained with copper, aluminium and tin powders have been summarized in the chart shown in Figure 10, which describes an initial characterization of the CS process applied to polymer substrates. Results obtained with copper had shown heavy erosion of the substrate, while aluminium only produced slight surface erosion but no deposition. On the other hand, tin has performed well and coatings were achieved on a variety of plastics over a range of parameters. As a consequence, the chart in Figure 10 has been divided into sections, each of them describing the probable deposition behaviour for the correspondent powder type. In this initial characterization chart, the zones boundaries are for simplicity straight lines. The chart reports the powder material, against the correspondent impact energy of particles travelling at optimum deposition speed. Values for the deposition/critical velocity of different materials and particle sizes can be calculated by the theoretical approach given by Schmidt [20]. For this case, a $25 \mu \mathrm{m}$ particle diameter was assumed, and the average velocity within the deposition window was selected for each material.

Dense and relatively strong particle materials, such as copper, can generate single energy impacts $E$ of nearly $0.02 \mathrm{~mJ}\left(E=1 / 2 m v^{2}\right)$, where $m$ is the particle mass and $v$ is the deposition

velocity. As particle sizes are in the order of microns for cold spray applications, this level of impact energy can result in severe contact stresses; therefore erosion of the polymer substrate is in all probability to occur. On the other hand, for lower strength metals such as tin, the critical velocity is most likely to diminish; therefore the impact energy transmitted on the substrate can dramatically decrease and deposition becomes achievable. It can be appreciated from Figure 10, that a particle of copper can generate about 10.7 times more energy at impact ${ }^{5}$ than tin. In contrast, light materials (such as aluminium), characterized by both low density and high deposition velocity, can generate negligible erosion levels on the substrate as the 

magnitudes, such as with gas-heating or Helium cold spray. A prediction of the behaviour of other metals, based upon the experimental results with aluminium, copper and tin powders, is included in Figure 10. Particles of stainless steel 316L would generate higher impact energy ${ }^{5}$ than copper, therefore erosion of the polymer substrate is in all probability to be predominant. Despite its high density, lead $(\mathrm{Pb})$ would only produce relatively low levels of impact energy resulting in possible deposition. On the other hand, titanium (Ti) places himself on the boundary within the transition window alongside aluminium. For these cases erosion may be negligible, however the critical velocity is high.

Figure 10

\section{4-CONCLUSIONS}

${ }^{1}$ Cold Spray (CS) is a relatively simple and inexpensive coating technique, which uses high speed gases in order to accelerate solid particles at high velocities. When impact with a substrate occurs, particles can bond together and form a coating. This technique has been widely used to obtain metal coatings onto surfaces of similar characteristics, such as other metals. This paper has investigated the compatibility of this process to produce metallic coatings onto surfaces such as polymers or composite materials. Powders for general engineering applications, such as commercial copper, aluminium, and tin have been sprayed onto a range of plastic materials (PC/ABS, polyamide-6, polypropylene, polystyrene) and a fibre-glass reinforced composite. Results have demonstrated that materials in the range of 
copper travelling at deposition velocities, can generate single particle impact energies in the order of $0.02 \mathrm{~mJ}$. This leads to severe contact stresses, therefore the predominant effect is erosion of the polymer. Aluminium, due to its low specific weight, does not bring any considerable damage to the surface; however its critical velocity could not be achieved with the cold spray system used in the experiments. On the other hand, it was possible to adjust the spraying parameters and select a suitable nozzle type to obtain coatings of tin on a variety of plastic substrate materials. ${ }^{3}$ The coating thickness was measured to be in the region between $45 \mu \mathrm{m}$ and nearly $100 \mu \mathrm{m}$, while the average critical velocity was estimated throughout a CFD analysis to be $310 \mathrm{~m} / \mathrm{s}$. The theoretical impact energy of tin powder was calculated to be 10.7 times lower ${ }^{5}$ than copper, resulting in negligible erosion. No surface pre-treatment was applied prior the spraying procedure, also coatings have shown good electrical conductivity. A process characterization chart has been therefore initialized, showing the relationship between properties of feedstock powders against deposition behaviour. By analyzing experimental results with aluminium, copper and tin, it was predicted that stainless steel 316L would cause erosion such as copper. On the other hand, titanium would perform similar to aluminium, and lead is most likely to successfully deposit.

\section{ACKNOWLEGMENTS}

The authors wish to express their gratitude to DTI/TSB, Pera, GKN, Hybrids, Haswell Mouldings, QPP, IFS, Dyne, for providing valuable industrial support and for contributing to the development of this work. 


\section{REFERENCES}

[1] L. Pawlowski, "The science and engineering of thermal spray coatings", Wiley, 2008.

[2] S. Deshpande, A. Kulkarni, S. Sampath, H. Herman, "Application of image analysis for characterization of porosity in thermal spray coatings and correlation with small angle neutron scattering”, Surface \& Coatings Technology, 187 (2004), 6-16.

[3] M.P. Planche, H. Liao, C. Coddet "Oxidation control in atmospheric plasma spraying coating", Surface \& Coatings Technology, 202 (2007), 69-76.

[4] V.V. Sobolev, J.Gilemany, J.M. Gilemany "HVOF spraying: theory and applications", The Institute of Materials, 1997.

[5] V.K. Champagne, "The cold spray material deposition process: fundamentals and applications", Woodhead Publishing Limited, 2007.

[6] Hong-Ren Wang, Bao-Rong Hou, Jun Wang, Qi Wang, and Wen-Ya Li, "Effect of process conditions on microstructure and corrosion resistance of cold-sprayed Ti Coatings", Journal of Thermal Spray Technology, Volume 17 (5-6), 2008, 736-741.

[7] E. Sansoucy, G.E. Kim, A.L. Moran, and B. Jodoin, "Mechanical characteristics of Al-CoCe coatings produced by the Cold Spray Process", Journal of Thermal Spray Technology, Volume 16 (5-6), 2007, 651-660.

[8] T. Marrocco, D.G. McCartney, P.H. Shipway, and A.J. Sturgeon, "Production of Titanium Deposits by Cold-Gas Dynamic Spray: Numerical Modeling and Experimental Characterization", Journal of Thermal Spray Technology, Volume 15 (2), 2006, 263-272.

[9] G. Bae, S. Kumar, Sanghoon Yoon, Kicheol Kang, Hyuntaek Na, Hyung-Jun Kim, Changhee Lee, "Bonding features and associated mechanisms in kinetic sprayed titanium coatings", Acta Materialia, 57 (2009), 5654-5666.

[10] Y. Xu, I.M. Hutchings, "Cold spray deposition of thermoplastic powder", Surface \& Coatings Technology, 201 (2006), 3044-3050.

[11] M. Bray, A. Cockburn, W. O’Neill "The laser assisted cold spray process and deposit characterization", Surface \& Coatings Technology, 203 (2009), 2851-2857. 
[12] Joel Voyer, Peter Schulz, and Martha Schreiber, "Electrically conductive flame sprayed aluminum coatings on textile substrates", Journal of Thermal Spray Technology, Volume 17 (5-6), 2008, 818-823.

[13] D. Zhang, P.H. Shipway, and D.G. McCartney, "Cold Gas Dynamic Spraying of Aluminum: The Role of Substrate Characteristics in Deposit Formation", Journal of Thermal Spray Technology, 14(1), March 2005, 109.

[14] Ha Yong Lee, Se Hun Jung, Soo Yong Lee, Young Ho You, Kyung Hyun Ko, "Correlation between $\mathrm{Al}_{2} \mathrm{O}_{3}$ particles and interface of $\mathrm{Al}-\mathrm{Al}_{2} \mathrm{O}_{3}$ coatings by cold spray", Applied Surface Science, 252 (2005), 1891-1898.

[15] Ha Yong Lee, Young Ho Yu, Young Cheol Lee, Young Pyo Hong, and Kyung Hyun Ko, "Cold Spray of $\mathrm{SiC}$ and $\mathrm{Al}_{2} \mathrm{O}_{3}$ with soft metal incorporation: A Technical Contribution", Journal of Thermal Spray Technology, Volume 13(2), June 2004, 184-189.

[16] A. Sturgeon, B. Dunn, S. Celotto, W.O’Neill, “Cold sprayed coatings for polymer composite substrate" European Space Agency, (Special Publication), ESA SP, n616, September 2006.

[17] Fluent 6.3.26 User Guide.

[18] R. Nickel, K. Bobzin, E. Lugscheider, D. Parkot, W. Varava, H. Olivier, X. Luo, "Numerical Studies of the Application of Shock Tube Technology for Cold Gas Dynamic Spray Process", Journal of Thermal Spray Technology, Volume 16 (5-6), 2005, 729-735.

[19] J. Vlcek, L. Gimeno, H. Huber, and E. Lugscheider, "A systematic approach to material eligibility for the Cold-Spray Process", Journal of Thermal Spray Technology, Volume 14 (1), 2005, 125-133.

[20] Tobias Schmidt, Hamid Assadi, Frank Gartner, Horst Richter, Thorsten Stoltenhoff, Heinrich Kreye, Thomas Klassen, "From Particle Acceleration to Impact and Bonding in Cold Spraying", Journal of Thermal Spray Technology, 18(5-6), Mid-December 2009, 794-808.

[21] Tobias Schmidt, Frank Gartner, Hamid Assadi, Heinrich Kreye, "Development of a generalized parameter window for cold spray deposition", Acta Materialia, 54 (2006), 729742 . 


\section{LIST OF FIGURE CAPTIONS}

Figure 1: The ${ }^{1}$ Cold Spray (CS) system.

Figure 2: SEM image of aluminium powder.

Figure 3: SEM picture of copper powder.

Figure 4: SEM picture of tin powder.

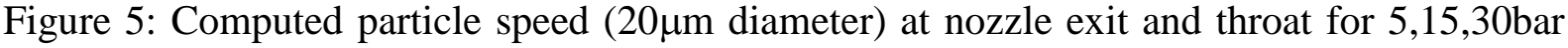
nozzle inlet pressure with nitrogen.

Figure 6: Close up pictures of copper tracks. (a), (b) on PC/ABS and glass-fibre reinforced composite with 5bar inlet pressure. (c), (d) 30bar inlet pressure.

Figure 7: Micrographs of track cross-section when spraying copper powder. (a),(b) low speed 5 bar nozzle inlet pressure onto PC/ABS and glass-fibre reinforced composite. (c), (d) high speed 30 bar inlet pressure onto PC/ABS and glass-fibre reinforced composite.

Figure 8: Tin tracks on various polymer substrates.

Figure 9: Micrographs of tin coatings cross-sections on a variety of polymer substrates.

Figure 10: ${ }^{1}$ Cold Spray (CS) process initial characterization chart for polymer substrates. 


\section{FIGURES}

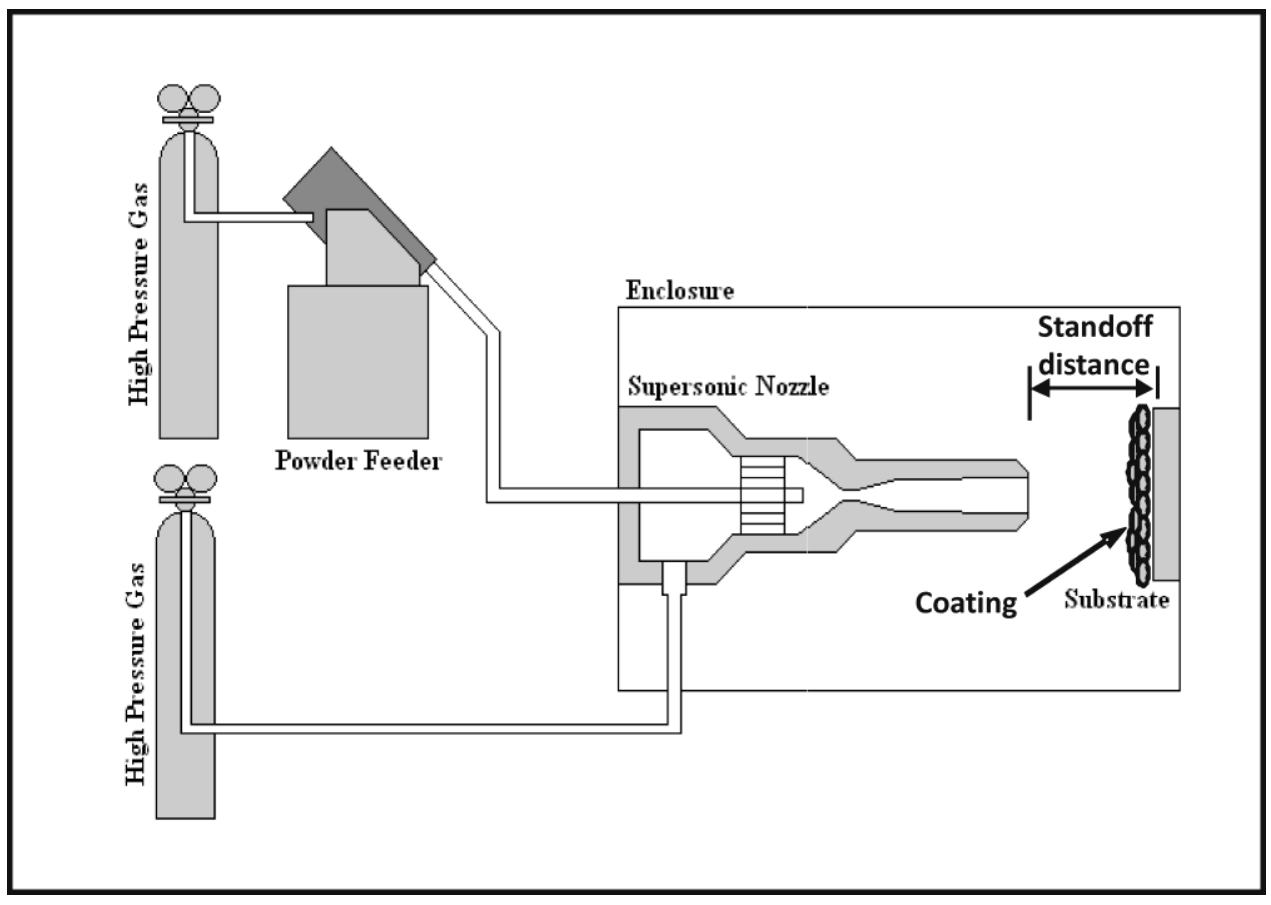

Figure 1 (modified according to Reviewer 2)

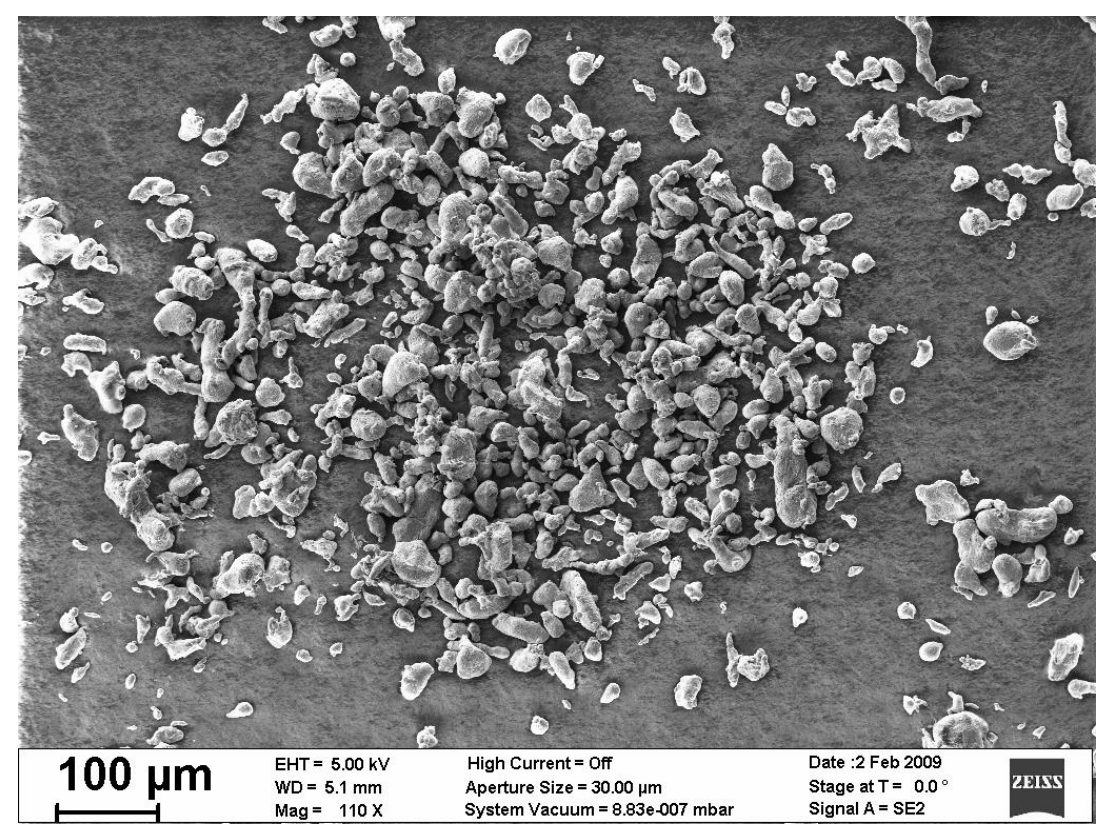

Figure 2 


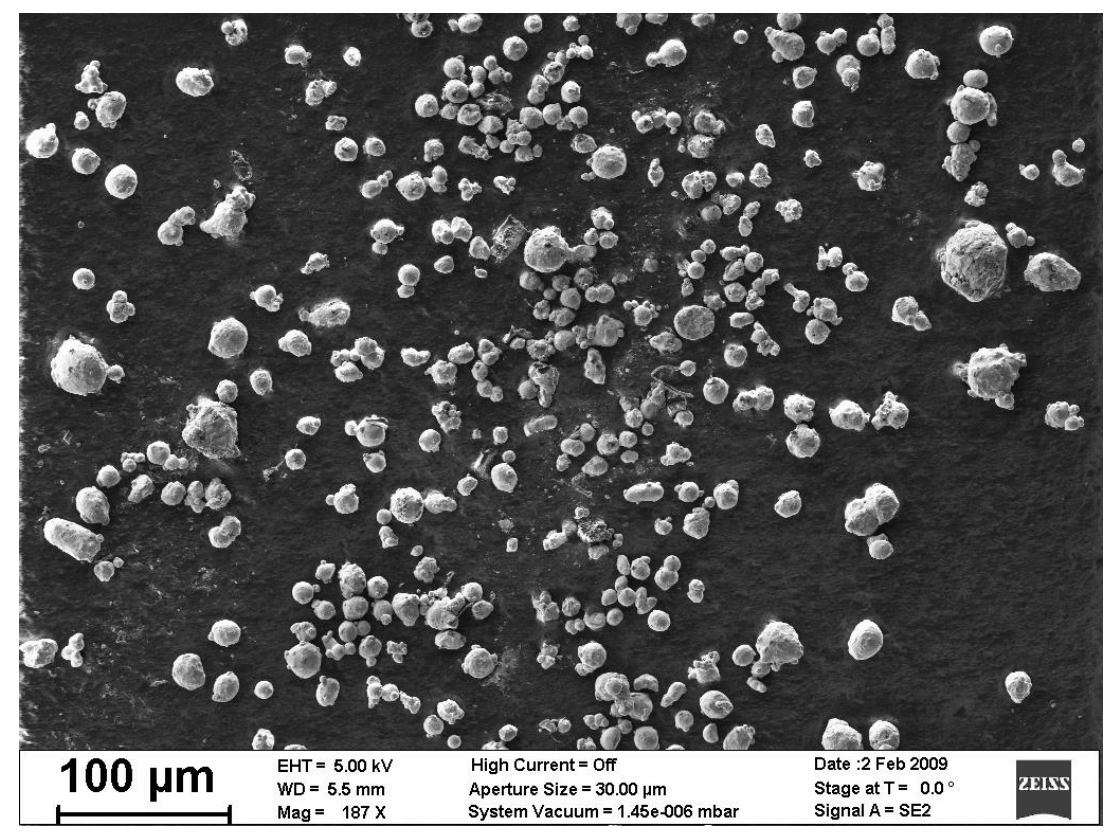

Figure 3

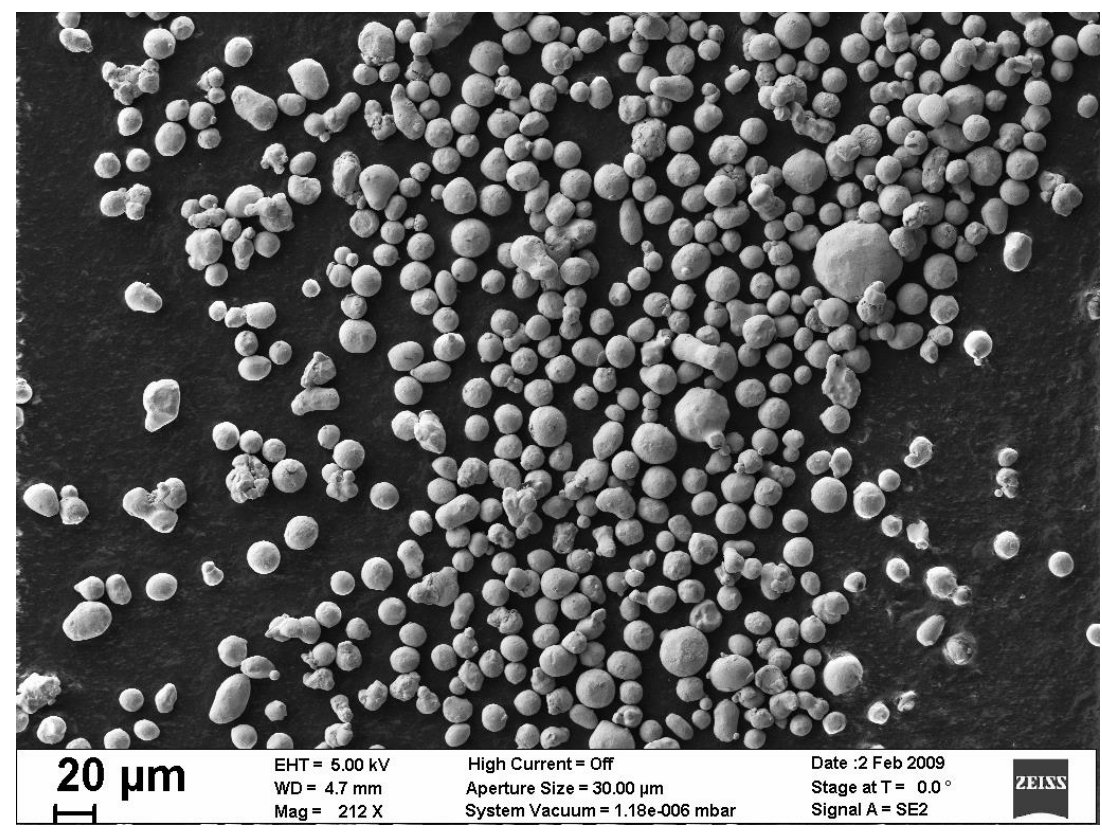

Figure 4 


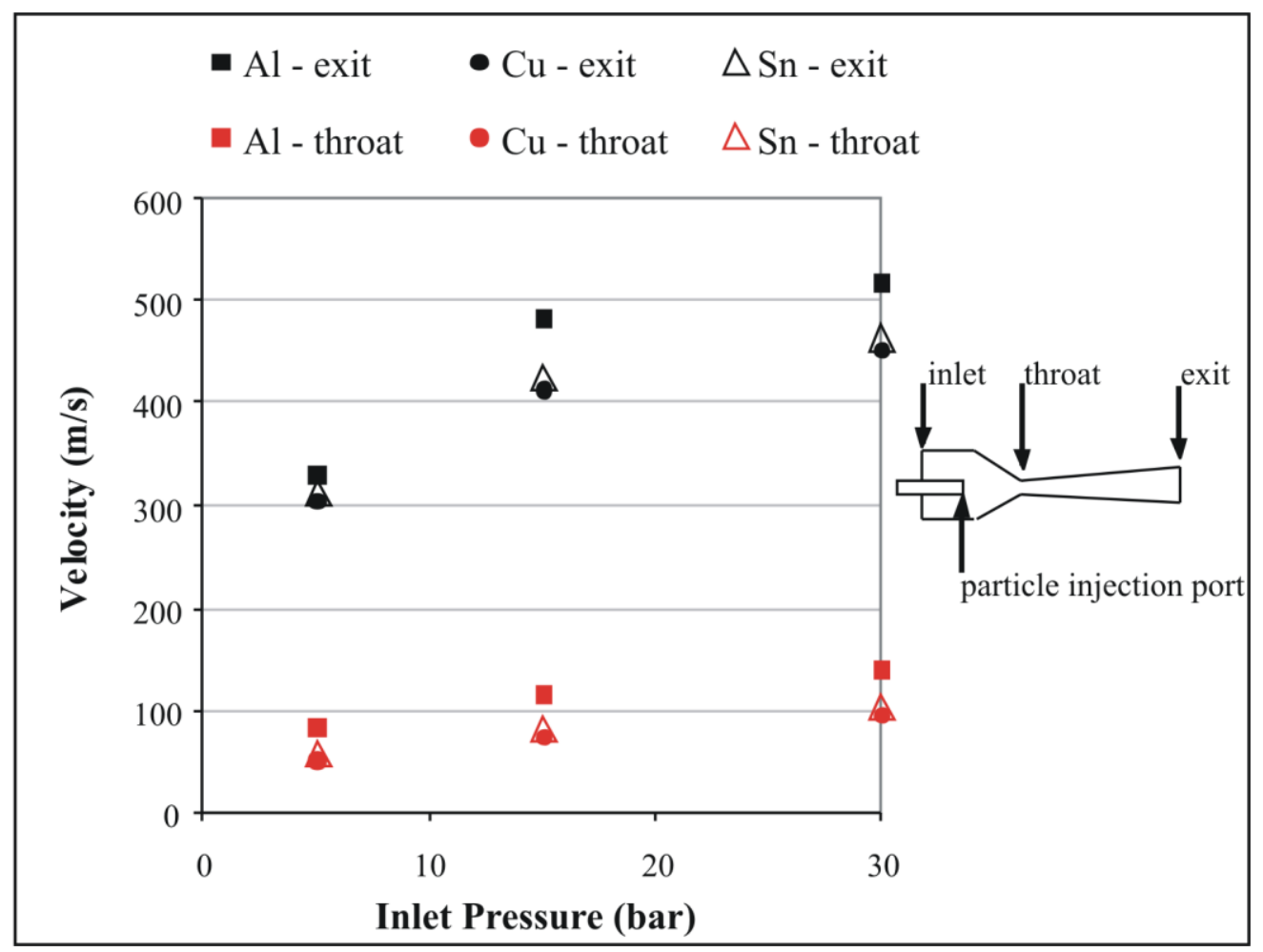

Figure 5 


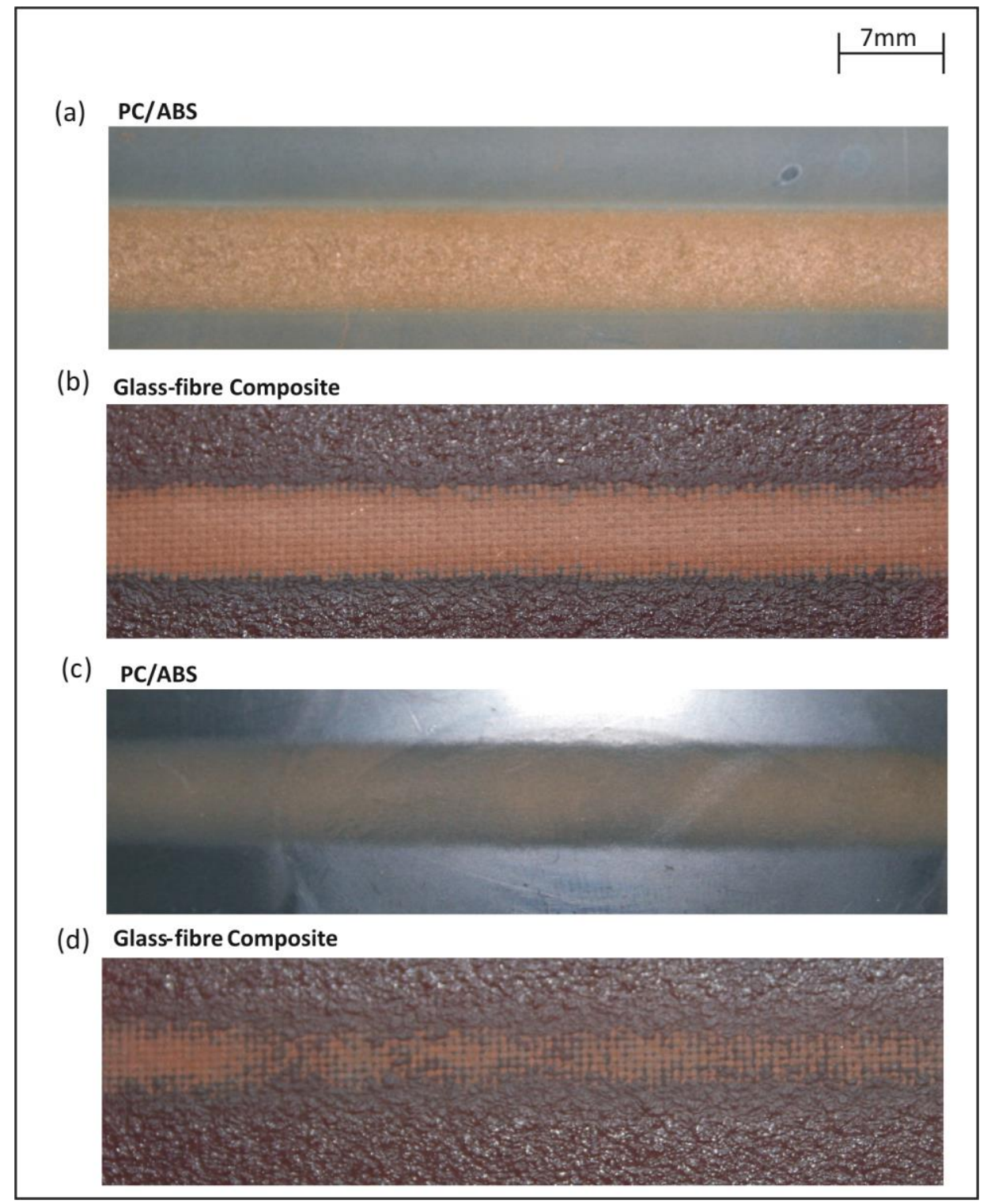

Figure 6 


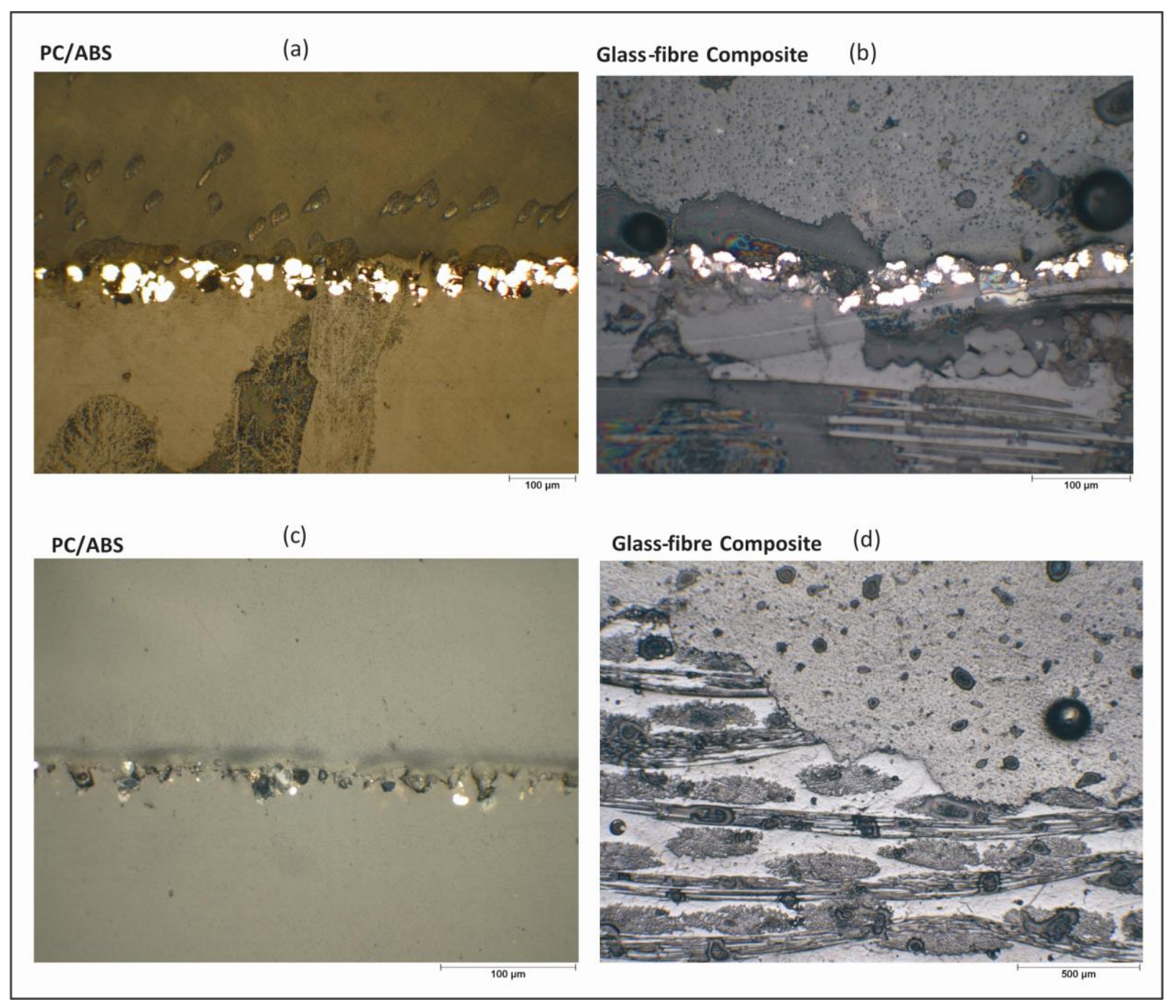

Figure 7

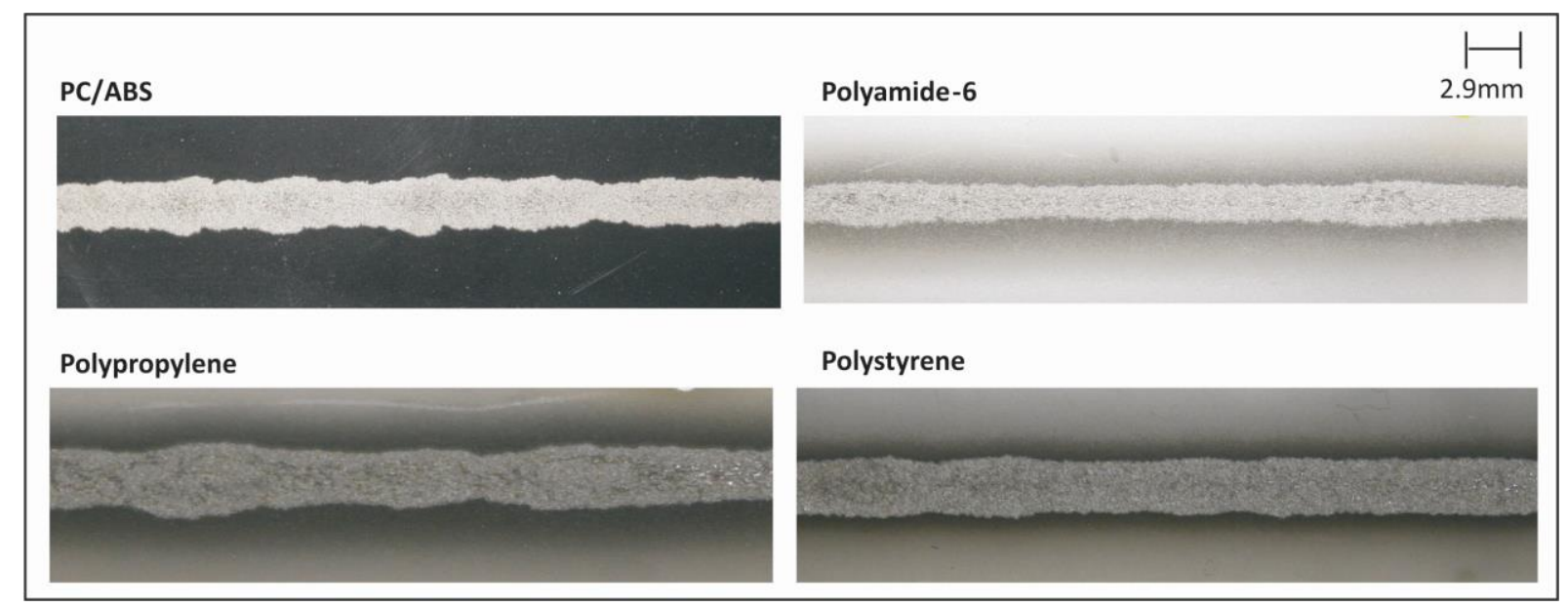

Figure 8 


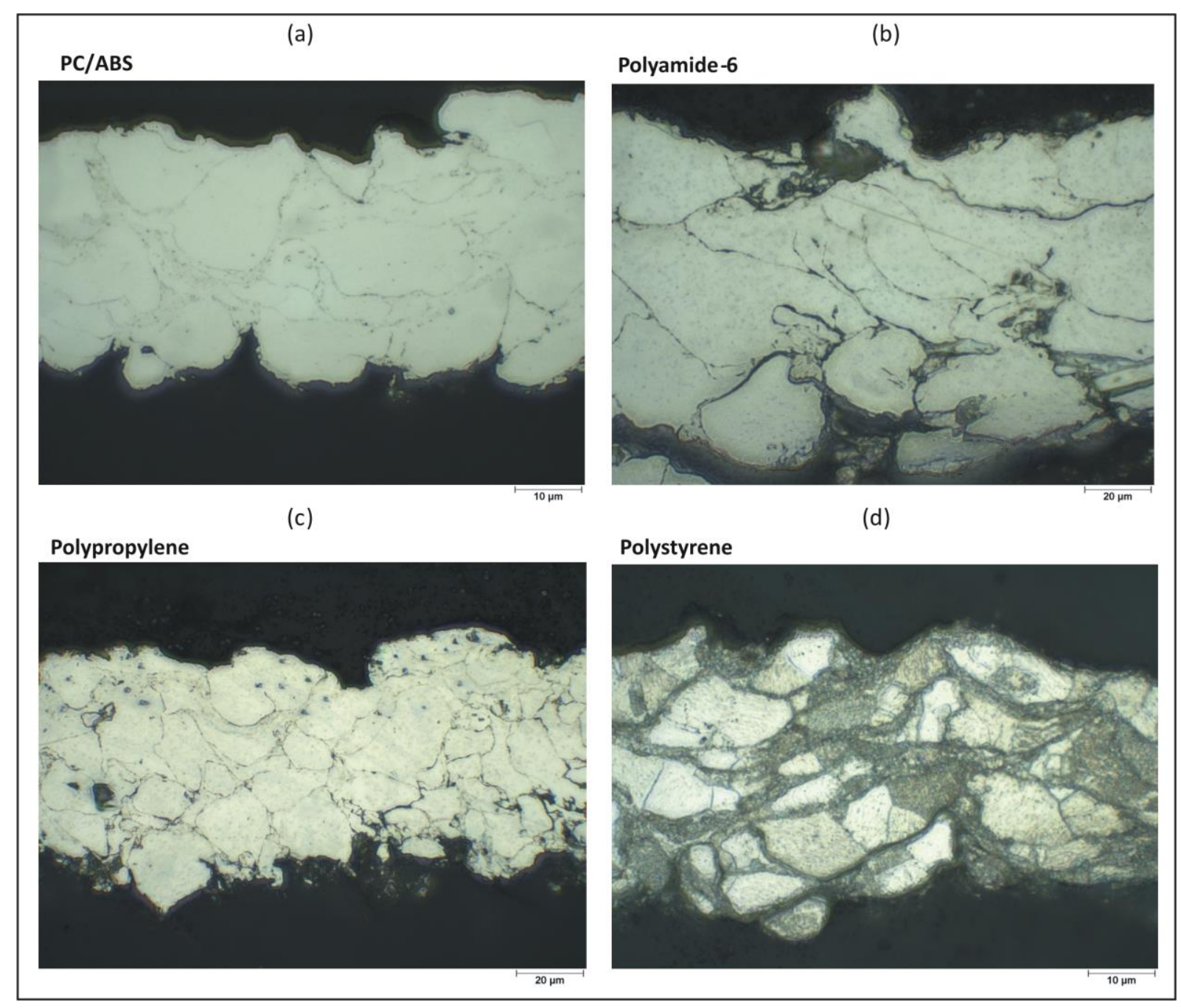

Figure 9 


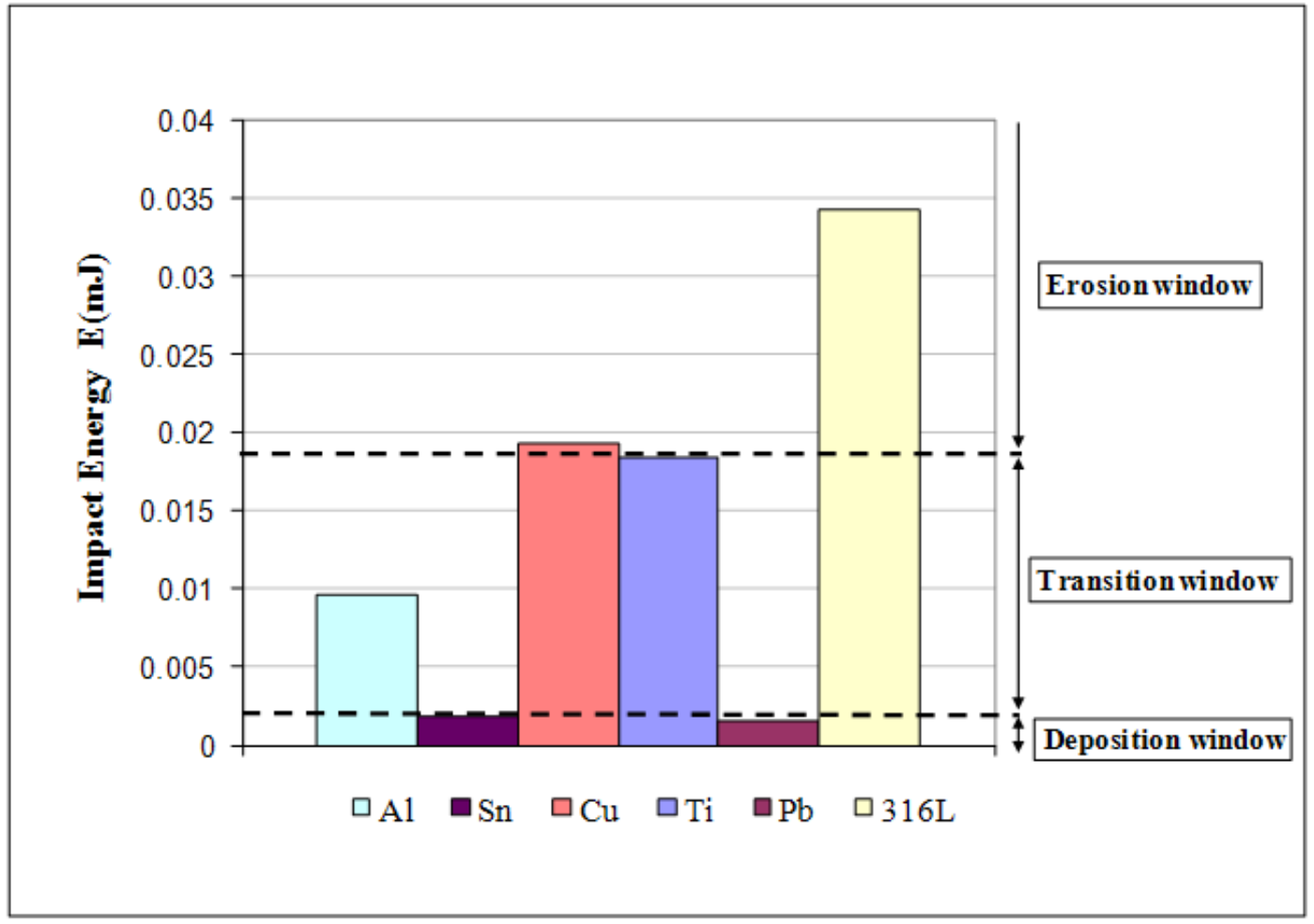

${ }^{4}$ Figure 10 (modified according to Reviewer 1) 